\title{
THE FIXED POINT PROPERTY IN DIRECT SUMS AND MODULUS $R(a, X)$
}

\author{
ANDRZEJ WIŚNICKI \\ (Received 30 October 2012; accepted 31 March 2013; first published online 28 June 2013)
}

\begin{abstract}
We show that the direct sum $\left(X_{1} \oplus \cdots \oplus X_{r}\right)_{\psi}$ with a strictly monotone norm has the weak fixed point property for nonexpansive mappings whenever $M\left(X_{i}\right)>1$ for each $i=1, \ldots, r$. In particular, $\left(X_{1} \oplus \cdots \oplus X_{r}\right)_{\psi}$ enjoys the fixed point property if Banach spaces $X_{i}$ are uniformly nonsquare. This combined with the earlier results gives a definitive answer for $r=2$ : a direct sum $X_{1} \oplus_{\psi} X_{2}$ of uniformly nonsquare spaces with any monotone norm has the fixed point property. Our results are extended to asymptotically nonexpansive mappings in the intermediate sense.
\end{abstract}

2010 Mathematics subject classification: primary 47H10; secondary 46B20, 47H09.

Keywords and phrases: nonexpansive mapping, asymptotically nonexpansive mapping, fixed point, direct sum.

\section{Introduction}

A Banach space $X$ is said to have the fixed point property (FPP) if every nonexpansive mapping $T: C \rightarrow C$, that is,

$$
\|T x-T y\| \leq\|x-y\|, \quad x, y \in C,
$$

acting on a (nonempty) bounded closed and convex subset $C$ of $X$ has a fixed point. A Banach space $X$ is said to have the weak fixed point property (WFPP) if we additionally assume that $C$ is weakly compact. The fixed point theorem of Kirk [19] asserts that every Banach space with weak normal structure has the WFPP. Recall that a Banach space $X$ has weak normal structure if $r(C)<\operatorname{diam} C$ for all weakly compact convex subsets $C$ of $X$ consisting of more than one point, where $r(C)=\inf _{x \in C} \sup _{y \in C}\|x-y\|$ is the Chebyshev radius of $C$. For more information regarding metric fixed point theory we refer the reader to $[1,14,21]$.

The permanence properties of normal structure and other conditions which guarantee the FPP under the direct sum operation have been studied extensively since the 1968 theorem of Belluce et al. [3], which states that a direct sum of two Banach

(c) 2013 Australian Mathematical Publishing Association Inc. 0004-9727/2013 \$16.00 
spaces with normal structure, endowed with the maximum norm, also has normal structure. Nowadays, there exist many results concerning permanence properties of conditions which imply normal structure (see [8] for a survey). However, the problem is more difficult if at least one of the spaces lacks weak normal structure (see [29] and references therein) and it is quite well understood only for nonexpansive mappings defined on rectangles $C_{1} \times C_{2}$ (see [20, 22]).

It was recently proved in [29] that if a Banach space $X$ has the WFPP and $Y$ has the generalised Gossez-Lami Dozo property (which is a slightly stronger property than weak normal structure) or is uniformly convex in every direction, then the direct sum $X \oplus Y$ with a strictly monotone norm has the WFPP. The present paper is concerned with direct sums $\left(X_{1} \oplus \cdots \oplus X_{r}\right)_{\psi}$ of Banach spaces with $M\left(X_{i}\right)>1$. (See Section 2 for the definitions of a $\psi$-direct sum, $M(X)$ and $R(a, X)$.) Dhompongsa et al. [7] proved that $\left(X_{1} \oplus \cdots \oplus X_{r}\right)_{\psi}$ has the WFPP whenever $M\left(X_{i}\right)>1$ for each $i=1, \ldots, r$ and $\psi \in \Psi_{r}$ is strictly convex. Kato and Tamura [17] proved that if $\psi \neq \psi_{1}$ then $R\left(X_{1} \oplus_{\psi} X_{2}\right)<2$ if and only if $R\left(X_{1}\right)<2$ and $R\left(X_{2}\right)<2$. Subsequently, Kato and Tamura [18] showed that $R\left(a,\left(X_{1} \oplus \cdots \oplus X_{r}\right)_{\infty}\right)=$ $\max _{1 \leq i \leq r} R\left(a, X_{i}\right)$ and, consequently, $\left(X_{1} \oplus \cdots \oplus X_{r}\right)_{\infty}$ has the WFPP whenever $M\left(X_{i}\right)>1$ for each $i$.

In this paper we show that $\left(X_{1} \oplus \cdots \oplus X_{r}\right)_{\psi}$ has the WFPP if $M\left(X_{i}\right)>1$ for each $i=1, \ldots, r$ and the norm $\|\cdot\|_{\psi}$ is strictly monotone. In particular, $\left(X_{1} \oplus \cdots \oplus X_{r}\right)_{\psi}$ has the FPP if Banach spaces $X_{i}$ are uniformly nonsquare and $\|\cdot\|_{\psi}$ is strictly monotone. This, combined with the aforementioned results, gives a definitive answer for $r=2$ : a direct sum $X_{1} \oplus_{\psi} X_{2}$ of uniformly nonsquare spaces with any monotone norm has the FPP. Theorems 3.8 and 3.9 extend our results to asymptotically nonexpansive mappings in the intermediate sense.

\section{Preliminaries}

The modulus $R(a, X)$ of a Banach space $X$ was defined by Domínguez Benavides [9] as a generalisation of the coefficient $R(X)$ introduced by García Falset [11]. Recall that, for a given $a \geq 0$,

$$
R(a, X)=\sup \left\{\liminf _{n \rightarrow \infty}\left\|x_{n}+x\right\|\right\},
$$

where the supremum is taken over all $x \in X$ with $\|x\| \leq a$ and all weakly null sequences in the unit ball $B_{X}$ such that

$$
D\left(\left(x_{n}\right)\right)=\limsup _{n \rightarrow \infty} \limsup _{m \rightarrow \infty}\left\|x_{n}-x_{m}\right\| \leq 1 .
$$

Define

$$
M(X)=\sup \left\{\frac{1+a}{R(a, X)}: a \geq 0\right\} .
$$

Then the condition $M(X)>1$ implies that $X$ has the WFPP for nonexpansive mappings (see [9]). We shall need the following characterisation of $M(X)$ proved in [5, Lemma 4.3] (see also [12, Corollary 4.3]). 
Lemma 2.1. Let X be a Banach space. The following conditions are equivalent:

(i) $\quad M(X)>1$;

(ii) there exists $a>0$ such that $R(a, X)<1+a$;

(iii) for every $a>0, R(a, X)<1+a$.

Let us now recall terminology concerning direct sums. A norm $\|\cdot\|$ on $\mathbb{R}^{n}$ is said to be monotone if

$$
\left\|\left(x_{1}, \ldots, x_{n}\right)\right\| \leq\left\|\left(y_{1}, \ldots, y_{n}\right)\right\|
$$

whenever $\left|x_{1}\right| \leq\left|y_{1}\right|, \ldots,\left|x_{n}\right| \leq\left|y_{n}\right|$. A norm $\|\cdot\|$ is said to be strictly monotone if

$$
\left\|\left(x_{1}, \ldots, x_{n}\right)\right\|<\left\|\left(y_{1}, \ldots, y_{n}\right)\right\|
$$

whenever $\left|x_{i}\right| \leq\left|y_{i}\right|$ for $i=1, \ldots, n$ and $\left|x_{i_{0}}\right|<\left|y_{i_{0}}\right|$ for some $i_{0}$. It is easy to see that $\ell_{p}^{n}$-norms, $1 \leq p<\infty$, are strictly monotone. A norm $\|\cdot\|$ on $\mathbb{R}^{n}$ is said to be absolute if

$$
\left\|\left(x_{1}, \ldots, x_{n}\right)\right\|=\left\|\left(\left|x_{1}\right|, \ldots,\left|x_{n}\right|\right)\right\|
$$

for every $\left(x_{1}, x_{2}, \ldots, x_{n}\right) \in \mathbb{R}^{n}$. It is well known (see [2]) that a norm is absolute if and only if it is monotone.

We will assume that the norm is normalised, that is,

$$
\|(1,0, \ldots, 0)\|=\cdots=\|(0, \ldots, 0,1)\|=1 .
$$

Bonsall and Duncan [6] showed that the set of all absolute and normalised norms on $\mathbb{R}^{2}$ $(\mathbb{C})$ is in one-to-one correspondence with the set $\Psi$ of all continuous convex functions on $[0,1]$ satisfying $\psi(0)=\psi(1)=1$ and $\max \{1-t, t\} \leq \psi(t) \leq 1$ for $0 \leq t \leq 1$, where the correspondence is given by

$$
\psi(t)=\|(1-t, t)\|, \quad 0 \leq t \leq 1 .
$$

Conversely, for any $\psi \in \Psi$ define

$$
\left\|\left(x_{1}, x_{2}\right)\right\|_{\psi}=\left(\left|x_{1}\right|+\left|x_{2}\right|\right) \psi\left(\frac{\left|x_{2}\right|}{\left|x_{1}\right|+\left|x_{2}\right|}\right)
$$

for $\left(x_{1}, x_{2}\right) \neq(0,0)$ and $\|(0,0)\|_{\psi}=0$. Then $\|\cdot\|_{\psi}$ is an absolute and normalised norm which satisfies $(2.1)$ (see $[6,24]$ ). For example, the $\ell_{p}^{2}$-norms correspond to the functions

$$
\psi_{p}(t)= \begin{cases}\left((1-t)^{p}+t^{p}\right)^{1 / p} & \text { if } 1 \leq p<\infty \\ \max \{1-t, t\} & \text { if } p=\infty\end{cases}
$$

It was proved in [27, Corollary 3] that a norm $\|\cdot\|_{\psi}$ in $\mathbb{R}^{2}$ is normalised and strictly monotone if and only if

$$
\psi(t)>\psi_{\infty}(t)
$$

for all $0<t<1$. 
Saito et al. [25] generalised the result of [6] to the $n$-dimensional case. Let

$$
\triangle_{n}=\left\{\left(s_{1}, \ldots, s_{n-1}\right) \in \mathbb{R}^{n-1}: s_{1}+\cdots+s_{n-1} \leq 1, s_{i} \geq 0, i=1, \ldots, n-1\right\}
$$

and denote by $\Psi_{n}$ the set of all continuous convex functions on $\Delta_{n}$ which satisfy the following conditions:

$$
\begin{gathered}
\psi(1,0, \ldots, 0)=\psi(0,1,0, \ldots, 0)=\cdots=\psi(0, \ldots, 0,1)=1 \\
\psi\left(s_{1}, \ldots, s_{n-1}\right) \geq\left(s_{1}+\cdots+s_{n-1}\right) \psi\left(\frac{s_{1}}{s_{1}+\cdots+s_{n-1}}, \ldots, \frac{s_{n-1}}{s_{1}+\cdots+s_{n-1}}\right), \\
\psi\left(s_{1}, \ldots, s_{n-1}\right) \geq\left(1-s_{1}\right) \psi\left(0, \frac{s_{2}}{1-s_{1}}, \ldots, \frac{s_{n-1}}{1-s_{1}}\right) \\
\vdots \\
\psi\left(s_{1}, \ldots, s_{n-1}\right) \geq\left(1-s_{n-1}\right) \psi\left(\frac{s_{1}}{1-s_{n-1}}, \ldots, \frac{s_{n-2}}{1-s_{n-1}}, 0\right) .
\end{gathered}
$$

Then the set of all absolute and normalised norms on $\mathbb{R}^{n}\left(\mathbb{C}^{n}\right)$ is in one-to-one correspondence with the set $\Psi_{n}$, where the correspondence is given by

$$
\psi\left(s_{1}, \ldots, s_{n-1}\right)=\left\|\left(1-\sum_{i=1}^{n-1} s_{i}, s_{1}, \ldots, s_{n-1}\right)\right\|, \quad\left(s_{1}, \ldots, s_{n-1}\right) \in \Delta_{n},
$$

and if $\psi \in \Psi_{n}$ then

$$
\begin{aligned}
\left\|\left(x_{1}, x_{2}, \ldots, x_{n}\right)\right\|_{\psi}= & \left(\left|x_{1}\right|+\left|x_{2}\right|+\cdots+\left|x_{n}\right|\right) \\
& \times \psi\left(\frac{\left|x_{2}\right|}{\left|x_{1}\right|+\left|x_{2}\right|+\cdots+\left|x_{n}\right|}, \ldots, \frac{\left|x_{n}\right|}{\left|x_{1}\right|+\left|x_{2}\right|+\cdots+\left|x_{n}\right|}\right),
\end{aligned}
$$

defined for $\left(x_{1}, x_{2}, \ldots, x_{n}\right) \neq(0,0, \ldots, 0),\|(0,0, \ldots, 0)\|_{\psi}=0$, is an absolute and normalised norm which satisfies (2.2) (see [25, Theorem 3.4]).

Let $X_{1}, \ldots, X_{r}$ be Banach spaces and $\psi \in \Psi_{r}$. Following [27], we shall write $\left(X_{1} \oplus\right.$ $\left.\cdots \oplus X_{r}\right)_{\psi}$ for the $\psi$-direct sum with the norm $\left\|\left(x_{1}, \ldots, x_{r}\right)\right\|_{\psi}=\left\|\left(\left\|x_{1}\right\|, \ldots,\left\|x_{r}\right\|\right)\right\|_{\psi}$, where $\left(x_{1}, \ldots, x_{r}\right) \in X_{1} \times \cdots \times X_{r}$.

\section{Fixed point theorems}

Let $T: C \rightarrow C$ be a nonexpansive mapping, where $C$ is a nonempty weakly compact convex subset of a Banach space $X$. By the Kuratowski-Zorn lemma, there exists a minimal (in the sense of inclusion) convex and weakly compact set $K \subset C$ which is invariant under $T$. Let $\left(x_{n}\right)$ be an approximate fixed point sequence for $T$ in $K$, that is, $\lim _{n \rightarrow \infty}\left\|T x_{n}-x_{n}\right\|=0$. The Goebel-Karlovitz lemma (see $\left.[13,15]\right)$ asserts that

$$
\lim _{n \rightarrow \infty}\left\|x_{n}-x\right\|=\operatorname{diam} K
$$

for every $x \in K$. 
Suppose now that $X_{1}, X_{2}, \ldots, X_{r}$ are Banach spaces, $\psi \in \Psi_{r}$ and $T: K \rightarrow K$ is a nonexpansive mapping acting on a weakly compact convex and minimal invariant subset $K$ of a direct sum $\left(X_{1} \oplus \cdots \oplus X_{r}\right)_{\psi}$. Suppose further that diam $K=1$ and $\left(w_{n}\right)=\left(\left(x_{n}^{(1)}, \ldots, x_{n}^{(r)}\right)\right)$ is an approximate fixed point sequence for $T$ in $K$ weakly converging to $(0, \ldots, 0) \in K$ such that $\lim _{n \rightarrow \infty}\left\|x_{n}^{(r)}\right\|=0$. The following construction (for $r=2$ ) was proposed in [29]. Fix an integer $k \geq 1$ and a sequence $\left(\varepsilon_{n}\right)$ in $(0,1)$. Since $\left\|T w_{n}-w_{n}\right\|_{\psi}$ and $\left\|x_{n}^{(r)}\right\|$ converge to 0 , we can choose $w_{n_{1}}$ in such a way that $\left\|T w_{n_{1}}-w_{n_{1}}\right\|_{\psi}<\varepsilon_{1}$ and $\left\|x_{n_{1}}^{(r)}\right\|<\varepsilon_{1}$. Let us put

$$
D_{1}^{1}=\left\{w_{n_{1}}\right\}, D_{2}^{1}=\operatorname{conv}\left(\left\{w_{n_{1}}, T w_{n_{1}}\right\}\right), \ldots, D_{k}^{1}=\operatorname{conv}\left(D_{k-1}^{1} \cup T\left(D_{k-1}^{1}\right)\right)
$$

where conv $A$ denotes the convex hull of $A$. We obtain a family $D_{1}^{1} \subset D_{2}^{1} \subset \cdots \subset D_{k}^{1}$ of relatively compact convex subsets of $K$. It follows from the Goebel-Karlovitz lemma and the relative compactness of $D_{k}^{1}$ that there exists $n_{2}>n_{1}$ such that $\left\|T w_{n_{2}}-w_{n_{2}}\right\|_{\psi}<$ $\varepsilon_{2},\left\|x_{n_{2}}^{(r)}\right\|<\varepsilon_{2}$ and $\left\|w_{n_{2}}-z\right\|_{\psi}>1-\varepsilon_{2}$ for all $z \in D_{k}^{1}$. Put

$$
D_{1}^{2}=\operatorname{conv}\left(\left\{w_{n_{1}}, w_{n_{2}}\right\}\right), \ldots, D_{k}^{2}=\operatorname{conv}\left(D_{k-1}^{2} \cup T\left(D_{k-1}^{2}\right)\right) .
$$

Again, we can find $n_{3}>n_{2}$ such that $\left\|T w_{n_{3}}-w_{n_{3}}\right\|_{\psi}<\varepsilon_{3},\left\|x_{n_{3}}^{(r)}\right\|<\varepsilon_{3}$ and $\left\|w_{n_{3}}-z\right\|_{\psi}>$ $1-\varepsilon_{3}$ for all $z \in D_{k}^{2}$. Put

$$
D_{1}^{3}=\operatorname{conv}\left(\left\{w_{n_{1}}, w_{n_{2}}, w_{n_{3}}\right\}\right), \ldots, D_{k}^{3}=\operatorname{conv}\left(D_{k-1}^{3} \cup T\left(D_{k-1}^{3}\right)\right) .
$$

Continuing in this fashion, we obtain by induction a subsequence $\left(w_{n_{j}}\right)$ of $\left(w_{n}\right)$ and a family $\left\{D_{j}^{i}\right\}_{1 \leq j \leq k, i \geq 1}$ of subsets of $K$ such that:

(i) $\left\|T w_{n_{i}}-w_{n_{i}}\right\|_{\psi}<\varepsilon_{i}$;

(ii) $\left\|x_{n_{i}}^{(r)}\right\|<\varepsilon_{i}$;

(iii) $\left\|w_{n_{i}}-z\right\|_{\psi}>1-\varepsilon_{i}$ for all $z \in D_{k}^{i-1}$ (where $D_{k}^{0}=\emptyset$ );

(iv) $D_{1}^{i}=\operatorname{conv}\left(\left\{w_{n_{1}}, w_{n_{2}}, \ldots, w_{n_{i}}\right\}\right)$;

(v) $D_{j+1}^{i}=\operatorname{conv}\left(D_{j}^{i} \cup T\left(D_{j}^{i}\right)\right)$,

for every $i \geq 1$ and $j=1, \ldots, k-1$ (see [29, Lemma 3.1]).

Furthermore (see [29, Lemma 3.2]), for every $i \geq 1, j=1, \ldots, k$ and $u \in D_{j}^{i+1}$, there exists $z \in D_{j}^{i}$ such that

$$
\|z-u\|_{\psi}+\left\|u-w_{n_{i+1}}\right\|_{\psi} \leq\left\|z-w_{n_{i+1}}\right\|_{\psi}+3(j-1) \varepsilon_{i+1} .
$$

We need the following simple observation regarding the set $D_{k}^{1}$.

Lemma 3.1. If $u=\left(y^{(1)}, \ldots, y^{(r)}\right) \in D_{k}^{1}$ then $\left\|y^{(r)}\right\|<k \varepsilon_{1}$.

Proof. We first show by induction that for every $j \in\{2, \ldots, k\}$ and for every $v \in D_{j}^{1}$,

$$
\left\|v-w_{n_{1}}\right\|_{\psi}<(j-1) \varepsilon_{1} .
$$

For $j=2, D_{2}^{1}=\operatorname{conv}\left(\left\{w_{n_{1}}, T w_{n_{1}}\right\}\right)$ and inequality (3.1) follows from the fact that $\left\|T w_{n_{1}}-w_{n_{1}}\right\|_{\psi}<\varepsilon_{1}$. 
Now fix $j \in\{2, \ldots, k-1\}$ and assume that for every $u \in D_{j}^{1},\left\|u-w_{n_{1}}\right\|_{\psi}<(j-1) \varepsilon_{1}$. Since

$$
D_{j+1}^{1}=\operatorname{conv}\left(D_{j}^{1} \cup T\left(D_{j}^{1}\right)\right),
$$

it is enough to show that $\left\|v-w_{n_{1}}\right\|_{\psi}<j \varepsilon_{1}$ for every $v \in T\left(D_{j}^{1}\right)$. Let $v=T u$ for some $u \in D_{j}^{1}$. Then

$$
\left\|v-w_{n_{1}}\right\|_{\psi} \leq\left\|T u-T w_{n_{1}}\right\|_{\psi}+\left\|T w_{n_{1}}-w_{n_{1}}\right\|_{\psi} \leq\left\|u-w_{n_{1}}\right\|_{\psi}+\varepsilon_{1}<j \varepsilon_{1},
$$

and the proof of (3.1) is complete.

Let $w_{n_{1}}=\left(x_{n_{1}}^{(1)}, \ldots, x_{n_{1}}^{(r)}\right), u=\left(y^{(1)}, \ldots, y^{(r)}\right) \in D_{k}^{1}$. It follows from (3.1) that

$$
\left\|y^{(r)}-x_{n_{1}}^{(r)}\right\| \leq\left\|u-w_{n_{1}}\right\|_{\psi}<(k-1) \varepsilon_{1}
$$

and consequently, since $\left\|x_{n_{1}}^{(r)}\right\|<\varepsilon_{1},\left\|y^{(r)}\right\|<k \varepsilon_{1}$.

Notice that if the norm $\|\cdot\|_{\psi}$ on $\mathbb{R}^{r}$ is strictly monotone then, for every $\varepsilon>0$, there exists $\delta(\varepsilon)>0$ such that if $\left(t^{(1)}, \ldots, t^{(r-1)}, t^{\prime}\right),\left(t^{(1)}, \ldots, t^{(r-1)}, t^{\prime \prime}\right)$ belong to the unit ball of $\left(\mathbb{R}^{r},\|\cdot\|_{\psi}\right)$ and

$$
\left\|\left(t^{(1)}, \ldots, t^{(r-1)}, t^{\prime}\right)\right\|_{\psi}<\left\|\left(t^{(1)}, \ldots, t^{(r-1)}, t^{\prime \prime}\right)\right\|_{\psi}+\delta(\varepsilon),
$$

then $\left|t^{\prime}\right|<\left|t^{\prime \prime}\right|+\varepsilon$. Indeed, otherwise, there exist $\varepsilon_{0}>0$ and two sequences $\left(\left(t_{n}^{(1)}, \ldots, t_{n}^{(r-1)}, t_{n}^{\prime}\right)\right),\left(\left(t_{n}^{(1)}, \ldots, t_{n}^{(r-1)}, t_{n}^{\prime \prime}\right)\right)$ in the unit ball such that

$$
\left\|\left(t_{n}^{(1)}, \ldots, t_{n}^{(r-1)}, t_{n}^{\prime}\right)\right\|_{\psi}<\left\|\left(t_{n}^{(1)}, \ldots, t_{n}^{(r-1)}, t_{n}^{\prime \prime}\right)\right\|_{\psi}+\frac{1}{n}
$$

and $\left|t_{n}^{\prime}\right| \geq\left|t_{n}^{\prime \prime}\right|+\varepsilon_{0}$. Passing to convergent subsequences and taking limits,

$$
\left\|\left(g^{(1)}, \ldots, g^{(r-1)}, g^{\prime}\right)\right\|_{\psi} \leq\left\|\left(g^{(1)}, \ldots, g^{(r-1)}, g^{\prime \prime}\right)\right\|_{\psi}
$$

and $\left|g^{\prime}\right| \geq\left|g^{\prime \prime}\right|+\varepsilon_{0}$, which contradicts the strict monotonicity of $\|\cdot\|_{\psi}$.

Thus, using Lemma 3.1, we can follow the arguments in [29, Lemma 3.3] and obtain the following result.

Lemma 3.2. Let $X_{1}, X_{2}, \ldots, X_{r}$ be Banach spaces, $\psi \in \Psi_{r}$, and $T: K \rightarrow K a$ nonexpansive mapping acting on a weakly compact convex and minimal invariant subset $K$ of a direct sum $\left(X_{1} \oplus \cdots \oplus X_{r}\right)_{\psi}$ with a strictly monotone norm $\|\cdot\|_{\psi}$. Suppose that $\operatorname{diam} K=1$ and $\left(w_{n}\right)=\left(\left(x_{n}^{(1)}, \ldots, x_{n}^{(r)}\right)\right)$ is an approximate fixed point sequence for $T$ in $K$ weakly converging to $(0, \ldots, 0) \in K$ such that $\lim _{n \rightarrow \infty}\left\|x_{n}^{(r)}\right\|=0$. Then for every positive integer $k$, there exist a sequence $\left(\varepsilon_{n}\right)$ in $(0,1)$, a subsequence $\left(w_{n_{j}}\right)$ of $\left(w_{n}\right)$ and a family $\left\{D_{j}^{i}\right\}_{1 \leq j \leq k, i \geq 1}$ of subsets of $K$ (depending on $k$ ) such that the above conditions (i)-(v) are satisfied and $\left\|y^{(r)}\right\|<1 / k$ for every $u=\left(y^{(1)}, \ldots, y^{(r)}\right) \in \bigcup_{i=1}^{\infty} D_{k}^{i}$.

We show that the appropriate approximate fixed point sequence $\left(w_{n}\right)$ exists if $M\left(X_{i}\right)>1, i=1, \ldots, r$. 
Lemma 3.3. Let $X_{1}, \ldots, X_{r}$ be Banach spaces with $M\left(X_{i}\right)>1$ for each $i=1, \ldots, r$, $\psi \in \Psi_{r}$, and $T: K \rightarrow K$ a nonexpansive mapping acting on a weakly compact convex and minimal invariant subset $K$ of a direct sum $\left(X_{1} \oplus \cdots \oplus X_{r}\right)_{\psi}$ with a strictly monotone norm $\|\cdot\|_{\psi}$. Suppose that diam $K=1$ and $\left(w_{n}\right)=\left(\left(x_{n}^{(1)}, \ldots, x_{n}^{(r)}\right)\right)$ is an approximate fixed point sequence for $T$ in $K$ weakly converging to $(0, \ldots, 0) \in K$. Then $\lim \inf _{n \rightarrow \infty}\left\|x_{n}^{\left(i_{0}\right)}\right\|=0$ for some $i_{0} \in\{1, \ldots, r\}$.

Proof. Let $\left(w_{n}\right)=\left(\left(x_{n}^{(1)}, \ldots, x_{n}^{(r)}\right)\right)$ be an approximate fixed point sequence for $T$ in $K$ weakly converging to $(0, \ldots, 0) \in K$ and suppose, contrary to our claim, that for each $i=1, \ldots, r, \lim \inf _{n \rightarrow \infty}\left\|x_{n}^{(i)}\right\|>0$. We can assume, passing to a subsequence, that

$$
d_{1}=\min \left\{\lim _{n \rightarrow \infty}\left\|x_{n}^{(1)}\right\|, \ldots, \lim _{n \rightarrow \infty}\left\|x_{n}^{(r)}\right\|\right\}>0 .
$$

We will show that for every $\varepsilon>0$ there exists $\delta_{1}(\varepsilon)>0$ such that if $v \in K$ and $\|T v-v\|_{\psi}<\delta_{1}(\varepsilon)$ then $\|v\|_{\psi}>1-\varepsilon$. Indeed, otherwise, arguing as in [10, Lemma 2], there exist $\varepsilon_{0}>0$ and a sequence $\left(v_{n}\right) \subset K$ such that $\left\|T v_{n}-v_{n}\right\|_{\psi}<1 / n$ and $\left\|v_{n}\right\|_{\psi} \leq 1-\varepsilon_{0}$ for every $n \in \mathbb{N}$. Thus $\left(v_{n}\right)$ is an approximate fixed point sequence in $K$, but lim $\sup _{n \rightarrow \infty}\left\|v_{n}\right\|_{\psi} \leq 1-\varepsilon_{0}$, which contradicts the Goebel-Karlovitz lemma since $(0, \ldots, 0) \in K$.

Furthermore, since $\left(\mathbb{R}^{r},\|\cdot\|_{\psi}\right)$ is finite-dimensional and the norm $\|\cdot\|_{\psi}$ is strictly monotone, for every $\eta>0$, there exists $\delta_{2}(\eta)>0$ such that if $\left(t^{(1)}, \ldots, t^{(r)}\right),\left(s^{(1)}, \ldots, s^{(r)}\right)$ belong to the unit ball, $\left|t^{(i)}\right| \leq\left|s^{(i)}\right|$ for $i=1, \ldots, r$ and $\left|t^{\left(i_{0}\right)}\right|<\left|s^{\left(i_{0}\right)}\right|-\eta$ for some $i_{0}$, then

$$
\left\|\left(t^{(1)}, \ldots, t^{(r)}\right)\right\|_{\psi}<\left\|\left(s^{(1)}, \ldots, s^{(r)}\right)\right\|_{\psi}-\delta_{2}(\eta) .
$$

Let

$$
A=\inf _{d_{1} / 6 r \leq a \leq 1} \min _{1 \leq i \leq r}\left(1+a-R\left(a, X_{i}\right)\right)
$$

and take $0<\eta<A d_{1} / 3, \varepsilon<\min \left\{\delta_{2}(\eta), d_{1} / 6\right\}$ (notice that $A>0$ by Lemma 2.1). Choose $t=1-\left(d_{1} / 3\right), \gamma<\min \left\{1, \delta_{1}(\varepsilon)\right\}$ and define the contraction $S_{n}: K \rightarrow K$ by

$$
S_{n} x=(1-\gamma) T x+\gamma t w_{n} .
$$

By the contractive mapping principle, for any $n \in \mathbb{N}$, there exists a unique fixed point $z_{n}=\left(y_{n}^{(1)}, \ldots, y_{n}^{(r)}\right)$ of $S_{n}$. We can assume, passing to a subsequence, that $\left(z_{n}\right)$ converges weakly to $z=\left(y^{(1)}, \ldots, y^{(r)}\right)$ and the limits $\lim _{n \rightarrow \infty}\left\|y_{n}^{(i)}\right\|, \lim _{n, m \rightarrow \infty, n \neq m}\left\|y_{n}^{(i)}-y_{m}^{(i)}\right\|$ exist for each $i=1, \ldots, r$ (see, for example, [26]).

It is not difficult to see that

$$
\left\|T z_{n}-z_{n}\right\|_{\psi} \leq \gamma<\delta_{1}(\varepsilon)
$$

and hence

$$
\left\|z_{n}\right\|_{\psi}>1-\varepsilon
$$


for every $n \in \mathbb{N}$. Furthermore, for every $n, m \in \mathbb{N}$,

$$
\left\|z_{n}-z_{m}\right\|_{\psi} \leq t
$$

and, from the weak lower semicontinuity of the norm,

$$
\left\|z_{n}-z\right\|_{\psi} \leq \liminf _{m \rightarrow \infty}\left\|z_{n}-z_{m}\right\|_{\psi} \leq t .
$$

Thus

$$
\|z\|_{\psi} \geq\left\|z_{n}\right\|_{\psi}-\left\|z_{n}-z\right\|_{\psi}>1-\varepsilon-t>\frac{d_{1}}{6}
$$

and, consequently, there exists $i_{0} \in\{1, \ldots, r\}$ such that

$$
\left\|y^{\left(i_{0}\right)}\right\|>\frac{d_{1}}{6 r}
$$

since otherwise

$$
\|z\|_{\psi} \leq\left\|y^{(1)}\right\|+\left\|y^{(2)}\right\|+\cdots+\left\|y^{(r)}\right\| \leq \frac{d_{1}}{6} .
$$

Furthermore,

$$
\left\|z_{n}-w_{n}\right\|_{\psi} \leq(1-\gamma)\left\|T z_{n}-T w_{n}\right\|_{\psi}+(1-\gamma)\left\|T w_{n}-w_{n}\right\|_{\psi}+\gamma(1-t)\left\|w_{n}\right\|_{\psi},
$$

which gives

$$
\left\|z_{n}-w_{n}\right\|_{\psi} \leq 1-t+\frac{(1-\gamma)}{\gamma}\left\|T w_{n}-w_{n}\right\|_{\psi} .
$$

It follows from the weak lower semicontinuity of the norm that, for each $i=1, \ldots, r$,

$$
\left\|y^{(i)}\right\| \leq \liminf _{n \rightarrow \infty}\left\|y_{n}^{(i)}-x_{n}^{(i)}\right\| \leq \liminf _{n \rightarrow \infty}\left\|z_{n}-w_{n}\right\|_{\psi} \leq 1-t=\frac{d_{1}}{3}
$$

and, from the triangle inequality,

$$
\left\|y_{n}^{(i)}-y^{(i)}\right\| \geq\left\|x_{n}^{(i)}\right\|-\left\|x_{n}^{(i)}-y_{n}^{(i)}\right\|-\left\|y^{(i)}\right\| .
$$

Hence

$$
\lim _{n \rightarrow \infty}\left\|y_{n}^{(i)}-y^{(i)}\right\| \geq \frac{d_{1}}{3}
$$

for each $i=1, \ldots, r$. Write

$$
d_{2}=\lim _{n, m \rightarrow \infty, n \neq m}\left\|y_{n}^{\left(i_{0}\right)}-y_{m}^{\left(i_{0}\right)}\right\|
$$

and notice that

$$
d_{2}=\limsup _{n \rightarrow \infty} \limsup _{m \rightarrow \infty}\left\|y_{n}^{\left(i_{0}\right)}-y_{m}^{\left(i_{0}\right)}\right\| \geq \lim _{n \rightarrow \infty}\left\|y_{n}^{\left(i_{0}\right)}-y^{\left(i_{0}\right)}\right\| \geq \frac{d_{1}}{3}
$$


by (3.3). It follows that

$$
\begin{aligned}
\lim _{n \rightarrow \infty}\left\|y_{n}^{\left(i_{0}\right)}\right\| & =\lim _{n \rightarrow \infty}\left\|\frac{y_{n}^{\left(i_{0}\right)}-y^{\left(i_{0}\right)}}{d_{2}}+\frac{y^{\left(i_{0}\right)}}{d_{2}}\right\| d_{2} \\
& \leq R\left(\frac{\left\|y^{\left(i_{0}\right)}\right\|}{d_{2}}, X_{i_{0}}\right) d_{2} \leq\left(1+\frac{\left\|y^{\left(i_{0}\right)}\right\|}{d_{2}}-A\right) d_{2} \\
& \leq d_{2}+\left\|y^{\left(i_{0}\right)}\right\|-A d_{2}<d_{2}+\left\|y^{\left(i_{0}\right)}\right\|-\eta
\end{aligned}
$$

since $d_{1} / 6 r \leq\left\|y^{\left(i_{0}\right)}\right\| / d_{2} \leq 1$. Therefore,

$$
\begin{aligned}
\lim _{n \rightarrow \infty}\left\|z_{n}\right\|_{\psi}< & \left\|\left(\lim _{n \rightarrow \infty}\left\|y_{n}^{(1)}\right\|, \ldots, d_{2}+\left\|y^{\left(i_{0}\right)}\right\|, \ldots, \lim _{n \rightarrow \infty}\left\|y_{n}^{(r)}\right\|\right)\right\|_{\psi}-\delta_{2}(\eta) \\
\leq & \left\|\left(\lim _{n \rightarrow \infty}\left\|y_{n}^{(1)}-y^{(1)}\right\|, \ldots, \lim _{n \rightarrow \infty}\left\|y_{n}^{(r)}-y^{(r)}\right\|\right)\right\|_{\psi} \\
& +\left\|\left(\left\|y^{(1)}\right\|, \ldots,\left\|y^{(r)}\right\|\right)\right\|_{\psi}-\varepsilon \\
\leq & \lim _{n, m \rightarrow \infty, n \neq m}\left\|z_{n}-z_{m}\right\|_{\psi}+\liminf _{n \rightarrow \infty}\left\|z_{n}-w_{n}\right\|_{\psi}-\varepsilon \\
\leq & t+1-t-\varepsilon \leq 1-\varepsilon
\end{aligned}
$$

which contradicts (3.2).

We can now formulate our main result. The proof combines the arguments of [29, Theorem 3.4] and Lemma 3.3.

Theorem 3.4. Let $X_{1}, \ldots, X_{r}$ be Banach spaces with $M\left(X_{i}\right)>1$ for each $i=1, \ldots, r$, $\psi \in \Psi_{r}$. Then the direct sum $\left(X_{1} \oplus \cdots \oplus X_{r}\right)_{\psi}$ with a strictly monotone norm $\|\cdot\|_{\psi}$ has the WFPP.

Proof. Assume that $\left(X_{1} \oplus \cdots \oplus X_{r}\right)_{\psi}$ does not have the WFPP. Then there exist a weakly compact convex subset $C$ of $\left(X_{1} \oplus \cdots \oplus X_{r}\right)_{\psi}$ and a nonexpansive mapping $T$ : $C \rightarrow C$ without a fixed point. By the Kuratowski-Zorn lemma, there exists a convex and weakly compact set $K \subset C$ which is minimal invariant under $T$ and which is not a singleton. Let $\left(w_{n}\right)=\left(\left(x_{n}^{(1)}, \ldots, x_{n}^{(r)}\right)\right)$ be an approximate fixed point sequence for $T$ in $K$. Without loss of generality we can assume that diam $K=1,\left(w_{n}\right)$ converges weakly to $(0, \ldots, 0) \in K$ and the limits $\lim _{n, m \rightarrow \infty, n \neq m}\left\|w_{n}-w_{m}\right\|_{\psi}, \lim _{n \rightarrow \infty}\left\|x_{n}^{(i)}\right\|, i=1, \ldots, r$, exist. Applying Lemma 3.3 gives $\lim _{n \rightarrow \infty}\left\|x_{n}^{\left(i_{0}\right)}\right\|=0$ for some $i_{0} \in\{1, \ldots, r\}$, and by rearrangement of Banach spaces we can assume that $i_{0}=r$. Now we follow the arguments in [29]. Lemma 3.2 shows that, for every positive integer $k$, there exist a sequence $\left(\varepsilon_{n}\right)$ in $(0,1)$, a subsequence $\left(w_{n_{j}}\right)$ of $\left(w_{n}\right)$ and a family $\left\{D_{j}^{i}\right\}_{1 \leq j \leq k, i \geq 1}$ of subsets of $K$ (depending on $k$ ) such that conditions (i) $-(\mathrm{v})$ are satisfied and $\left\|y^{(r)}\right\|<1 / k$ for every $u=\left(y^{(1)}, \ldots, y^{(r)}\right) \in \bigcup_{i=1}^{\infty} D_{k}^{i}$.

Let $C_{0}=\{(0, \ldots, 0)\}$ and $C_{j}=\operatorname{conv}\left(C_{j-1} \cup T\left(C_{j-1}\right)\right)$ for $j \geq 1$. It is not difficult to see that $\operatorname{cl}\left(\bigcup_{j=1}^{\infty} C_{j}\right)$ is a closed convex subset of $K$ which is invariant for $T$ (and hence equals $K)$. Fix $k \geq 1$ and notice that $(0, \ldots, 0) \in \operatorname{cl}\left(\cup_{i=1}^{\infty} D_{1}^{i}\right)$, because the 
sequence $\left(w_{n_{j}}\right)_{j \geq 1}$ converges weakly to $(0, \ldots, 0)$. Furthermore, for $j<k$,

$$
T\left(\operatorname{cl}\left(\bigcup_{i=1}^{\infty} D_{j}^{i}\right)\right)=\operatorname{cl}\left(\bigcup_{i=1}^{\infty} T\left(D_{j}^{i}\right)\right) \subset \operatorname{cl}\left(\bigcup_{i=1}^{\infty} D_{j+1}^{i}\right)
$$

and hence, by induction on $j$,

$$
C_{j} \subset \operatorname{cl}\left(\bigcup_{i=1}^{\infty} D_{j+1}^{i}\right) \subset \mathrm{cl}\left(\bigcup_{i=1}^{\infty} D_{k}^{i}\right), \quad j<k .
$$

It follows that if $\left(y^{(1)}, \ldots, y^{(r)}\right) \in C_{j}$ and $j<k$, then $\left(y^{(1)}, \ldots, y^{(r)}\right) \in \operatorname{cl}\left(\bigcup_{i=1}^{\infty} D_{k}^{i}\right)$ and, consequently, $\left\|y^{(r)}\right\| \leq 1 / k$. Letting $k \rightarrow \infty$, we have $y^{(r)}=0$ for every $\left(y^{(1)}, \ldots, y^{(r)}\right) \in$ $\operatorname{cl}\left(\cup_{j=1}^{\infty} C_{j}\right)=K$. Therefore, $K$ is a subset of $\left(X_{1} \oplus \cdots \oplus X_{r-1} \oplus\{0\}\right)_{\psi}$ which is isometric to $\left(X_{1} \oplus \cdots \oplus X_{r-1}\right)_{\psi^{\prime}}$ with the strictly monotone norm $\left\|\left(x_{1}, \ldots, x_{r-1}\right)\right\|_{\psi^{\prime}}=$ $\left\|\left(x_{1}, \ldots, x_{r-1}, 0\right)\right\|_{\psi}$. Repeating the above procedure $r-1$ times, we deduce that $K$ is isometric to some $X_{j_{0}}, j_{0} \in\{1, \ldots, r\}$. Since $M\left(X_{j_{0}}\right)>1, T$ has a fixed point in $K$, which contradicts our assumption.

García Falset et al. [12] introduced the modulus $R W(a, X)$, which plays an important role in fixed point theory for nonexpansive mappings. For a given $a \geq 0$,

$$
R W(a, X)=\sup \min \left\{\liminf _{n}\left\|x_{n}+x\right\|, \liminf _{n}\left\|x_{n}-x\right\|\right\},
$$

where the supremum is taken over all $x \in X$ with $\|x\| \leq a$ and all weakly null sequences in the unit ball $B_{X}$. Let

$$
M W(X)=\sup \left\{\frac{1+a}{R W(a, X)}: a \geq 0\right\} .
$$

It was proved in [12, Theorem 3.3] that $M(X) \geq M W(X)$ whenever $B_{X^{*}}$ is $w^{*}$ sequentially compact. Since a minimal invariant set $K$ is always separable, we obtain the following corollary.

Corollary 3.5. Let $X_{1}, \ldots, X_{r}$ be Banach spaces with $M W\left(X_{i}\right)>1$ for each $i=$ $1, \ldots, r, \psi \in \Psi_{r}$. Then the direct sum $\left(X_{1} \oplus \cdots \oplus X_{r}\right)_{\psi}$ with a strictly monotone norm $\|\cdot\|_{\psi}$ has the WFPP.

Recall that a Banach space $X$ is uniformly nonsquare if

$$
J(X)=\sup _{x, y \in S_{X}} \min \{\|x+y\|,\|x-y\|\}<2 .
$$

Corollary 4.4 in [12] shows that if $X$ is uniformly nonsquare, then $M W(X)>1$. Since uniformly nonsquare spaces are reflexive, the FPP and WFPP coincide and thus we obtain the following corollary.

Corollary 3.6. Let $X_{1}, \ldots, X_{r}$ be uniformly nonsquare Banach spaces, $\psi \in \Psi_{r}$. Then the direct sum $\left(X_{1} \oplus \cdots \oplus X_{r}\right)_{\psi}$ with a strictly monotone norm $\|\cdot\|_{\psi}$ has the FPP.

In the case $r=2$ we have a stronger, definitive result. 
Theorem 3.7. Let $X_{1}, X_{2}$ be uniformly nonsquare Banach spaces, $\psi \in \Psi_{2}$. Then the direct sum $X_{1} \oplus_{\psi} X_{2}$ has the FPP.

Proof. Kato et al. [16, Theorem 1] proved that $X_{1} \oplus_{\psi} X_{2}$ is uniformly nonsquare if and only if $X_{1}, X_{2}$ are uniformly nonsquare and $\psi \neq \psi_{1}, \psi_{\infty}$ (see also [4]). Kato and Tamura [17, Theorem 3.6] showed that $R\left(X_{1} \oplus_{\infty} X_{2}\right)<2$ and hence $X_{1} \oplus_{\infty} X_{2}$ has the FPP. The case $X_{1} \oplus_{1} X_{2}$ is covered by Corollary 3.6, since the norm $\|\cdot\|_{\psi_{1}}$ is strictly monotone.

We conclude with two fixed point theorems for asymptotically nonexpansive mappings. Recall that a mapping $T: C \rightarrow C$ is said to be asymptotically nonexpansive in the intermediate sense if $T$ is continuous and

$$
\limsup _{n \rightarrow \infty} \sup _{x, y \in C}\left(\left\|T^{n} x-T^{n} y\right\|-\|x-y\|\right) \leq 0 .
$$

A Banach space $X$ is said to have the super fixed point property for nonexpansive mappings (asymptotically nonexpansive mappings) if every Banach space $Y$ which is finitely representable in $X$ has the FPP for nonexpansive mappings (asymptotically nonexpansive mappings).

Theorem 2.4 in [30] shows that $X$ has the super fixed point property for nonexpansive mappings if and only if $X$ has the super fixed point property for asymptotically nonexpansive mappings in the intermediate sense. Since the direct sum $\left(X_{1} \oplus \cdots \oplus X_{r}\right)_{\psi}$ of uniformly nonsquare spaces is stable under passing to the Banach space ultrapowers, it follows from the properties of ultrapowers and Corollary 3.6 (Theorem 3.7) that $\left(X_{1} \oplus \cdots \oplus X_{r}\right)_{\psi}$ with a strictly monotone norm $\left(X_{1} \oplus_{\psi} X_{2}\right.$ with any monotone norm) has the super fixed property for nonexpansive mappings and, consequently, for asymptotically nonexpansive mappings in the intermediate sense. Thus we obtain the following theorem.

Theorem 3.8. Let $X_{1}, \ldots, X_{r}$ be uniformly nonsquare Banach spaces, $\psi \in \Psi_{r}$. Then the direct sum $\left(X_{1} \oplus \cdots \oplus X_{r}\right)_{\psi}$ with a strictly monotone norm $\|\cdot\|_{\psi}$ has the (super) fixed point property for asymptotically nonexpansive mappings in the intermediate sense. The assumption about the strict monotonicity of the norm can be dropped if $r=2$.

Recall that [28, Theorem 2.3] shows that the direct sum $\left(X_{1} \oplus \cdots \oplus X_{r}\right)_{\psi}$ of uniformly noncreasy spaces with a strictly monotone norm has the FPP. Since uniformly noncreasy spaces are stable under passing to the Banach space ultrapowers (see [23]), the conclusion of Theorem 3.8 is also valid in this case.

Theorem 3.9. Let $X_{1}, \ldots, X_{r}$ be uniformly noncreasy Banach spaces, $\psi \in \Psi_{r}$. Then the direct sum $\left(X_{1} \oplus \cdots \oplus X_{r}\right)_{\psi}$ with a strictly monotone norm $\|\cdot\|_{\psi}$ has the super fixed point property for asymptotically nonexpansive mappings in the intermediate sense. 


\section{Acknowledgement}

The author wishes to express his thanks to Tomás Domínguez Benavides for fruitful conversations regarding the subject of this paper.

\section{References}

[1] J. M. Ayerbe Toledano, T. Domínguez Benavides and G. López Acedo, Measures of Noncompactness in Metric Fixed Point Theory (Birkhäuser, Basel, 1997).

[2] F. L. Bauer, J. Stoer and C. Witzgall, 'Absolute and monotonic norms', Numer. Math. 3 (1961), 257-264.

[3] L. P. Belluce, W. A. Kirk and E. F. Steiner, 'Normal structure in Banach spaces', Pacific J. Math. 26 (1968), 433-440.

[4] A. Betiuk-Pilarska and S. Prus, 'Uniform nonsquareness of direct sums of Banach spaces', Topol. Methods Nonlinear Anal. 34 (2009), 181-186.

[5] A. Betiuk-Pilarska and A. Wiśnicki, 'On the Suzuki nonexpansive-type mappings', Ann. Funct. Anal. 4(2) (2013), 72-86.

[6] F. F. Bonsall and J. Duncan, Numerical Ranges. II (Cambridge University Press, Cambridge, 1973).

[7] S. Dhompongsa, A. Kaewcharoen and A. Kaewkhao, 'Fixed point property of direct sums', Nonlinear Anal. 63 (2005), e2177-e2188.

[8] S. Dhompongsa and S. Saejung, 'Geometry of direct sums of Banach spaces', Chamchuri J. Math. 2 (2010), 1-9.

[9] T. Domínguez Benavides, 'A geometrical coefficient implying the fixed point property and stability results', Houston J. Math. 22 (1996), 835-849.

[10] T. Domínguez Benavides, 'A renorming of some nonseparable Banach spaces with the fixed point property', J. Math. Anal. Appl. 350 (2009), 525-530.

[11] J. García Falset, 'Stability and fixed points for nonexpansive mappings', Houston J. Math. 20 (1994), 495-506.

[12] J. García Falset, E. Lloréns Fuster and E. M. Mazcuñan Navarro, 'Uniformly nonsquare Banach spaces have the fixed point property for nonexpansive mappings', J. Funct. Anal. 233 (2006), 494-514.

[13] K. Goebel, 'On the structure of minimal invariant sets for nonexpansive mappings', Ann. Univ. Mariae Curie-Skłodowska Sect. A 29 (1975), 73-77.

[14] K. Goebel and W. A. Kirk, Topics in Metric Fixed Point Theory (Cambridge University Press, Cambridge, 1990).

[15] L. A. Karlovitz, 'Existence of fixed points of nonexpansive mappings in a space without normal structure', Pacific J. Math. 66 (1976), 153-159.

[16] M. Kato, K.-S. Saito and T. Tamura, 'Uniform non-squareness of $\psi$-direct sums of Banach spaces $X \oplus_{\psi} Y$ ', Math. Inequal. Appl. 7 (2004), 429-437.

[17] M. Kato and T. Tamura, 'Weak nearly uniform smoothness and worth property of $\psi$-direct sums of Banach spaces', Comment. Math. Prace Mat. 46 (2006), 113-129.

[18] M. Kato and T. Tamura, 'Uniform non- $\ell_{1}^{n}$-ness of $\ell_{\infty}$-sums of Banach spaces', Comment. Math. 49 (2009), 179-187.

[19] W. A. Kirk, 'A fixed point theorem for mappings which do not increase distances', Amer. Math. Monthly 72 (1965), 1004-1006.

[20] W. A. Kirk and C. Martinez-Yanez, 'Nonexpansive and locally nonexpansive mappings in product spaces', Nonlinear Anal. 12 (1988), 719-725.

[21] W. A. Kirk and B. Sims (eds.) Handbook of Metric Fixed Point Theory (Kluwer Academic Publishers, Dordrecht, 2001).

[22] T. Kuczumow, 'Fixed point theorems in product spaces', Proc. Amer. Math. Soc. 108 (1990), $727-729$. 
[23] S. Prus, 'Banach spaces which are uniformly noncreasy', in: Proc. 2nd World Congress of Nonlinear Analysts, Nonlinear Anal., 30 (ed. V. Lakshmikantham) (1997), 2317-2324.

[24] K.-S. Saito, M. Kato and Y. Takahashi, 'Von Neumann-Jordan constant of absolute normalized norms on $\mathbb{C}^{2}$, J. Math. Anal. Appl. 244 (2000), 515-532.

[25] K.-S. Saito, M. Kato and Y. Takahashi, 'Absolute norms on $\mathbb{C}^{n}$, J. Math. Anal. Appl. 252 (2000), 879-905.

[26] B. Sims and M. A. Smyth, 'On some Banach space properties sufficient for weak normal structure and their permanence properties', Trans. Amer. Math. Soc. 351 (1999), 497-513.

[27] Y. Takahashi, M. Kato and K.-S. Saito, 'Strict convexity of absolute norms on $\mathbb{C}^{2}$ and direct sums of Banach spaces', J. Inequal. Appl. 7 (2002), 179-186.

[28] A. Wiśnicki, 'Products of uniformly noncreasy spaces', Proc. Amer. Math. Soc. 130 (2002), 3295-3299.

[29] A. Wiśnicki, 'On the fixed points of nonexpansive mappings in direct sums of Banach spaces', Studia Math. 207 (2011), 75-84.

[30] A. Wiśnicki, 'The super fixed point property for asymptotically nonexpansive mappings', Fund. Math. 217 (2012), 265-277.

ANDRZEJ WIŚNICKI, Institute of Mathematics,
Maria Curie-Skłodowska University, 20-031 Lublin, Poland
e-mail: awisnic@ @ektor.umcs.lublin.pl 